@ 2002 American Chemical Society, Org. Lett., Bunnage ol0269704 Supporting Info Page 1

\title{
Synthesis of the Putative Structure of (-)-Oryzoxymycin
}

Mark E. Bunnage, ${ }^{\mathrm{b}}$ Thota Ganesh, ${ }^{\mathrm{a}}$ Ishmael B. Masesane, ${ }^{\mathrm{a}}$ Darren Orton ${ }^{\mathrm{a}}$, Patrick G.

\section{Steel ${ }^{a^{*}}$}

a Department of Chemistry, University of Durham, South Road, Durham, DH1 3LE, UK

${ }^{\mathrm{b}}$ Pfizer Global Research and Development, Sandwich, Kent, CT13 9NJ

p.g.steel@durham.ac.uk

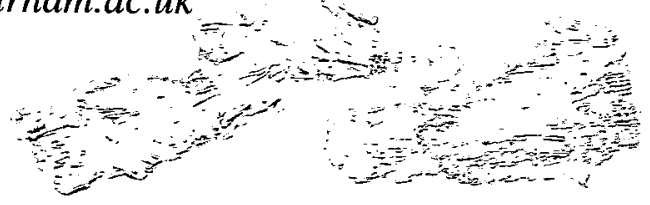

\section{Experimental Section}

All new stable compounds have satisfactory spectral and analytical data. Yields refer to pure isolated products.

Ethyl (+)-6-tert-butoxycarbonylamino-5-hydroxy-1,3-cyclohexadiene-1-carboxylate 10a To a solution of KHMDS (530 mg, $2.64 \mathrm{mmol})$ in THF $\left(10 \mathrm{~cm}^{3}\right)$ at $-50^{\circ} \mathrm{C}$ was added a solution of ester (-)-9a $(250 \mathrm{mg}, 0.88 \mathrm{mmol})$ in THF $\left(2.5 \mathrm{~cm}^{3}\right)$. The solution was then warmed up to room temperature (15 minutes) and quenched with a mixture of ethyl acetate and ethanol $\left(50 \mathrm{~cm}^{3}, 19: 1\right)$. The mixture was washed with sat. $\mathrm{NH}_{4} \mathrm{Cl}\left(25 \mathrm{~cm}^{3}\right)$, dried $\left(\mathrm{MgSO}_{4}\right)$ and concentrated under reduced pressure. The resulting residue was purified by flash chromatography eluting with petroleum ether/ethyl acetate mixture (7:3) to give cyclohexadiene $10 \mathrm{a}(178 \mathrm{mg}, 71 \%)$ as a pale yellow semi solid. $[\alpha]_{\mathrm{D}}^{21}+266(\mathrm{c}=$ $1, \mathrm{CHCl}_{3}$ ). Found: $\mathrm{C}, 59.20, \mathrm{H}, 7.53 ; \mathrm{N}, 4.80 . \mathrm{C}_{14} \mathrm{H}_{21} \mathrm{NO}_{5}$ requires $\mathrm{C}, 59.35, \mathrm{H}, 7.47, \mathrm{~N}$, 4.94. $v_{\max }(\mathrm{KBr}): 3400(\mathrm{br}), 2979,2931,1716,1700,1584,1514,1392,1366,1245,1169$, $1079,1017,915,737 \mathrm{~cm}^{-1} ; \delta_{\mathrm{H}}\left(500 \mathrm{MHz}, \mathrm{CDCl}_{3}\right): 1.28\left(3 \mathrm{H}, \mathrm{t}, \mathrm{J}=7.0 \mathrm{~Hz}, \mathrm{OCH}_{2} \mathrm{CH}_{3}\right), 1.42$ $\left(9 \mathrm{H}, \mathrm{s}, \mathrm{OC}\left(\mathrm{CH}_{3}\right)_{3}\right), 4.20\left(2 \mathrm{H}, \mathrm{q}, \mathrm{J}=7.0 \mathrm{~Hz}, \mathrm{OCH}_{2} \mathrm{CH}_{3}\right), 4.36(1 \mathrm{H}, \mathrm{s}, \mathrm{H}-5), 4.48(1 \mathrm{H}, \mathrm{br}$, $\mathrm{NH}), 4.76(1 \mathrm{H}, \mathrm{m}, \mathrm{H}-6), 6.26(2 \mathrm{H}, \mathrm{m}, \mathrm{H}-3$ and 4$), 7.17(1 \mathrm{H}, \mathrm{d}, \mathrm{J}=4.8 \mathrm{~Hz}) ; \delta_{\mathrm{c}}(125 \mathrm{MHz}$, $\left.\mathrm{CDCl}_{3}\right): 14.1\left(\mathrm{OCH}_{2} \mathrm{CH}_{3}\right), 28.3\left(\mathrm{OC}\left(\mathrm{CH}_{3}\right)_{3}\right), 50.0(\mathrm{C}-6), 60.8\left(\mathrm{OCH}_{2} \mathrm{CH}_{3}\right), 67.7(\mathrm{C}-5), 80.0$ $\left(\mathrm{OC}\left(\mathrm{CH}_{3}\right)_{3}\right), 124.5(\mathrm{C}-3), 132.6(\mathrm{C}-2), 133.5(\mathrm{C}-4), 155.4\left(\mathrm{NCO}_{2}\right), 165.9\left(\mathrm{CO}_{2} \mathrm{C}_{2} \mathrm{H}_{5}\right) ; \mathrm{m} / \mathrm{z}$ (CI): $284\left(\mathrm{MH}^{+}\right)$. The major by-product of the reaction was ethyl 2-hydroxybenzoate identified by comparison with an authentic sample. ${ }^{1}$ 
(C) 2002 American Chemical Society, Org. Lett., Bunnage ol0269704 Supporting Info Page 2

tert-Butyl (2'S,5R,6R)-(+)-2'-[6-tert-butoxycarbornylamino-5-hydroxycyclohexa-1,3diene-1-carbonyloxy]propionate 16a

$\mathrm{CsF}$ ( $240 \mathrm{mg}, 1.57 \mathrm{mmol}$ ) was dried under vacuum at $150^{\circ} \mathrm{C}$ for 5 hours. A suspension of the dried $\mathrm{CsF}$ in DMF $\left(5 \mathrm{~cm}^{3}\right)$ was stirred at $25^{\circ} \mathrm{C}$ for 30 minutes. To this suspension was added acid $15 \mathrm{a}$ ( $200 \mathrm{mg}, 0.78 \mathrm{mg}$ ) and the mixture was stirred at $25^{\circ} \mathrm{C}$ for 30 minutes. $(R)-\mathrm{MsOCH}\left(\mathrm{CH}_{3}\right) \mathrm{CO}_{2}{ }^{\mathrm{B}} \mathrm{Bu}$ was then added and the reaction mixture was stirred at $50^{\circ} \mathrm{C}$. After 24 hours the reaction mixture was partitioned between ethyl acetate $\left(3 \times 50 \mathrm{~cm}^{3}\right)$ and water $\left(50 \mathrm{~cm}^{3}\right)$. The combined organic extracts were washed with brine, dried $\left(\mathrm{MgSO}_{4}\right)$ and concentrated under reduced pressure to give a yellow oil. Purification by column chromatography eluting with petrol/ethyl acetate (6:4) gave 16a as a yellow gum (205 mg, 68\%); $[\alpha]_{\mathrm{D}}^{21.6}-171\left(\mathrm{c} 1, \mathrm{CHCl}_{3}\right) ; \delta_{\mathrm{H}}\left(500 \mathrm{MHz}, \mathrm{CDCl}_{3}\right): 1.43$ and $1.46(18 \mathrm{H}, s, 2$ x $\left.\mathrm{OC}\left(\mathrm{CH}_{3}\right)_{3}\right), 1.51(3 \mathrm{H}, d, \mathrm{~J}=6.9 \mathrm{~Hz}, \mathrm{H}-3), 2.90(1 \mathrm{H}, \mathrm{br}, \mathrm{OH}), 4.55(1 \mathrm{H}, \mathrm{br}, \mathrm{H}-5$ '), 4.76 $\left.\left(1 \mathrm{H}, m, \mathrm{H}-6^{\prime}\right), 5.061 \mathrm{H}, q, \mathrm{~J}=6.9 \mathrm{~Hz}, \mathrm{H}-2\right), 6.30\left(2 \mathrm{H}, m, \mathrm{H}-3^{\prime}\right.$ and $\left.4^{\prime}\right), 7.22(1 \mathrm{H}, d, \mathrm{~J}=5.5$ $\left.\mathrm{Hz}, \mathrm{H}-2^{\prime}\right) ; \delta_{\mathrm{C}}\left(125 \mathrm{MHz}, \mathrm{CDCl}_{3}\right): 17.1(\mathrm{C}-3), 28.2$ and $\left.28.42 \times \mathrm{OC}\left(\mathrm{CH}_{3}\right)_{3}\right), 51.2\left(\mathrm{C}-6^{\prime}\right)$, 68.9 (C-5'), 69.4 (C-2), 80.2 and $80.4\left(2 \times \mathrm{OC}\left(\mathrm{CH}_{3}\right)_{3}\right), 125.3$ (C-3'), $\left.125.3(\mathrm{C}-1 ')^{\prime}\right), 133.5$ (C-4'), $152.1\left(\mathrm{NCO}_{2}\right), 169.4$ and $170.1\left(2 \times \mathrm{CO}_{2}\right) ; \mathrm{MS} \mathrm{m} / \mathrm{z}\left(\mathrm{ES}^{+}\right): 406(\mathrm{MNa})^{+}$.

tert-Butyl (2'S,5S,6S)-(+)-2'-[6-tert-butoxycarbornylamino-5-hydroxycyclohexa-1,3diene-1-carbonyloxy]propionate $\mathbf{1 6 b}$

Acid 15b when subjected to the same conditions as above gave ester $16 \mathbf{b}$ as white gum white solid (89\%). (204 mg, 68\%); $[\alpha]_{\mathrm{D}}^{21.6}+181\left(\mathrm{c} 1, \mathrm{CHCl}_{3}\right) ; \delta_{\mathrm{H}}\left(500 \mathrm{MHz}, \mathrm{CDCl}_{3}\right): 1.43$ and $\left.1.46\left(18 \mathrm{H}, s, 2 \times \mathrm{OC}_{\left(\mathrm{CH}_{3}\right.}\right)_{3}\right), 1.49\left(3 \mathrm{H}, d, \mathrm{~J}=6.9 \mathrm{~Hz}, \mathrm{H}-3^{\prime}\right), 2.78(1 \mathrm{H}, \mathrm{br}, \mathrm{OH}), 4.40$ (1H, br, NH), 4.52 (1H, br, H-5), $\left.4.78(1 \mathrm{H}, m, \mathrm{H}-6), 5.04(1 \mathrm{H}, q, \mathrm{~J}=6.9 \mathrm{~Hz}, \mathrm{H}-2)^{\prime}\right), 6.27$ $(2 \mathrm{H}, m, \mathrm{H}-3$ and 4$), 7.24(1 \mathrm{H}, d, \mathrm{~J}=5.5 \mathrm{~Hz}, \mathrm{H}-2) ; \delta_{\mathrm{c}}\left(125 \mathrm{MHz}, \mathrm{CDCl}_{3}\right): 17.2\left(\mathrm{C}-3^{\prime}\right), 28.2$ and $28.6\left(2 \times \mathrm{OC}\left(\mathrm{CH}_{3}\right)_{3}\right), 50.6(\mathrm{C}-6), 68.4(\mathrm{C}-5), 69.7(\mathrm{C}-2), 80.2$ and $82.3(2 \mathrm{x}$ $\left.\mathrm{O} \underline{\mathrm{C}}\left(\mathrm{CH}_{3}\right)_{3}\right), 124.6(\mathrm{C}-3), 125.2(\mathrm{C}-1), 133.5(\mathrm{C}-4), 134.5(\mathrm{C}-2), 152\left(\mathrm{NCO}_{2}\right), 170.0$ and $170.1\left(\mathrm{CO}_{2}\right) ; \mathrm{m} / \mathrm{z}\left(\mathrm{ES}^{+}\right): 406(\mathrm{MNa})^{+}$.

(2'S,5R,6R) 2'-[6-amino-5-hydroxy-1,3-cyclohexadiene-1-carbonyloxy]propionic acid • trifluoroacetic acid salt (ent-'oryzoxymycin' trifluoroacetic acid salt) 17a: 
(C) 2002 American Chemical Society, Org. Lett., Bunnage ol0269704 Supporting Info Page 3

To a solution of ester $16 \mathbf{a}(140 \mathrm{mg}, 0.37 \mathrm{mmol})$ in dichloromethane $\left(5 \mathrm{~cm}^{3}\right)$ was added trifluoroacetic acid $(112 \mu \mathrm{l}, 1.46 \mathrm{mmol})$. This mixture was then stirred until all the starting material was consumed (TLC ca. $12 \mathrm{~h}$ ). The solvent was removed under reduced pressure to give a brown gum which was dissolved in water $\left(20 \mathrm{~cm}^{3}\right)$ and washed with ethyl acetate $\left(20 \mathrm{~cm}^{3}\right)$. The aqueous phase was concentrated under reduced pressure to the title salt $17 \mathrm{~b}$ as a yellow gum $(75 \mathrm{mg}, 89 \%) ;[\alpha]_{D}^{20.8}-199$ (c 1, $\left.\mathrm{H}_{2} \mathrm{O}\right) ; \mathrm{HRMS}\left(\mathrm{ES}^{+}\right)$Found $\mathrm{M}^{+}-\mathrm{H}_{2} \mathrm{O}, 209.0690 . \mathrm{C}_{10} \mathrm{H}_{11} \mathrm{NO}_{4}$ requires $M, 209.0688 ; v_{\max }(\mathrm{KBr}): 3413,3550-2800(\mathrm{br})$, 1726 (shoulder), 1679, 1580, 1271, 1202, 1137, 1095, $1044 \mathrm{~cm}^{-1} \delta_{\mathrm{H}}\left(500 \mathrm{MHz}, \mathrm{D}_{2} \mathrm{O}\right)$ : $1.57(3 \mathrm{H}, d, \mathrm{~J}=7.2 \mathrm{~Hz}, \mathrm{H}-3), 4.42$ (1H, $\left.m, \mathrm{H}-6^{\prime}\right), 4.45$ (1H, $\left.d, \mathrm{~J}=1.2 \mathrm{~Hz}, \mathrm{H}-5^{\prime}\right), 5.16$ $(1 \mathrm{H}, q, \mathrm{~J}=7.2 \mathrm{~Hz}, \mathrm{H}-2), 6.49\left(2 \mathrm{H}, m, \mathrm{H}-3^{\prime}\right.$ and $\left.\mathrm{H}-4^{\prime}\right), 7.57\left(1 \mathrm{H}, d, \mathrm{~J}=1.5 \mathrm{~Hz}, \mathrm{H}-2^{\prime}\right) ; \delta_{\mathrm{c}}$ (125 MHz, D 20 ): 16.4 (C-3), 48.4 (C-6'), 63.9 (C-5'), 71.2 (C-2), 121.2 (C-1'), 125.3 (3'), 132.5 (C-4'), 138.8 (C-2'), $166.2\left(\mathrm{CO}_{2}\right), 176.3$ (C-1), MS m/z (CI): $228\left(\mathrm{MH}^{+}\right)$.

(2'S,5S,6S) 2'-[6-amino-5-hydroxy-1,3-cyclohexadiene-1-carbonyloxy]propionic acid • trifluoroacetic acid salt 17b:

Ester 16b when subjected to the same conditions as above gave acid $16 \mathbf{b}$ as white solid (89\%); m.p. $106-108^{\circ} \mathrm{C},[\alpha]_{\mathrm{D}}{ }^{21}+165\left(\mathrm{c}=1, \mathrm{CHCl}_{3}\right)$. Found: C, 46.60, H, 5.34; N, 4.64. $\mathrm{C}_{10} \mathrm{H}_{13} \mathrm{NO}_{5} . \mathrm{C}_{2} \mathrm{HO}_{2} \mathrm{~F}_{3}$ requires $\mathrm{C}, 46.61, \mathrm{H}, 4.96, \mathrm{~N}, 4.53 . \mathrm{v}_{\max }(\mathrm{KBr}): 3425,3550-2800(\mathrm{br})$, $2927,1703,1677,1589,1276,1201,1138,1099,1044 \mathrm{~cm}^{-1} ; \delta_{\mathrm{H}}\left(500 \mathrm{MHz}, \mathrm{D}_{2} \mathrm{O}\right): 1.55$ $(3 \mathrm{H}, d, \mathrm{~J}=7.0 \mathrm{~Hz}, \mathrm{H}-3), 4.39(1 \mathrm{H}, d, \mathrm{~J}=\mathrm{H}-6), 4.43(1 \mathrm{H}, d d, \mathrm{~J}=3.0$ and $1.5 \mathrm{~Hz}, \mathrm{H}-5)$, $5.18\left(1 \mathrm{H}, q, \mathrm{~J}=7.0 \mathrm{~Hz}, \mathrm{H}-2{ }^{\prime}\right), 6.47(2 \mathrm{H}, m, \mathrm{H}-3$ and 4$), 7.55(1 \mathrm{H}, d, \mathrm{~J}=5.5 \mathrm{~Hz}, \mathrm{H}-2) ; \delta_{\mathrm{C}}$ (125 MHz, $\left.\mathrm{D}_{2} \mathrm{O}\right): 16.2\left(\mathrm{C}-3{ }^{\prime}\right), 48.5$ (C-6), 63.9 (C-5), 70.5 (C-2'), 121.0 (C-1), 125.3 (C3), $132.8(\mathrm{C}-4), 139.1(\mathrm{C}-2), 166.1\left(\mathrm{CO}_{2} \mathrm{H}\right), 175.3(\mathrm{C}=\mathrm{O}) ; \mathrm{m} / \mathrm{z}\left(\mathrm{CI} / \mathrm{NH}_{3}\right): 228\left(\mathrm{MH}^{+}\right.$, $100 \%), 210(96), 138(42)$.

(1) Perkins, M. V.; Kitching, W.; Drew, R. A. I.; Moore, C. J.; Konig, W. A. J. Chem. Soc., Perkin Trans. 1 1990, 1111-1117. 